\title{
La violencia contra niños, niñas y adolescentes, la línea entre el abuso y la disciplina.
}

\section{Violence against children and adolescents, the line between abuse and discipline.}

Teresa Paredes. ${ }^{1}$, Álvaro Jiménez. ${ }^{2}$, Malena Quiroga. ${ }^{3} \&$ Patricio Poaquiza. ${ }^{4}$

Recibido: 02-12-2017 / Revisado: 07-02-2018 Aceptado:15-03-2018/ Publicado: 01-04-2018

\begin{abstract}
.
DOI: https://doi.org/10.33262/cienciadigital.v2i2.79
\end{abstract}

Purpose: The present results of a Ambato Technical University research on violence against children and adolescents centered in the way parents, children and leaders understand and conceptualize violence. The paper explores how families communicate, the parental skills and the influence of community leaders.

Methods: The Project was done in the location of Pelileo-Ecuador, considered rural area, with a population of $(n=260), 59$ community leaders, 110 children and adolescents and 101 parents. The individuals lived in Pelileo, Bolivar, Huambalo y Cotalo small rural towns that belonged to the state of Tunguahua, Ecuador. A quantitative research using the survey for each group. The survey was validated in a research realized in Africa. The survey had open and closed questions and covered the following areas: disciplines styles, communication, and the cooperation of community leaders. Students from the Social Work career cooperated taken the survey, same that were trained in the process of the research, to warrant the good application of the survey, which took about ten minutes for each participant. The data was analyzed with SPSS23 (version windows 32 bits) The participants were informed and their participation was voluntary. The research followed the norms and principles

1 Universidad Técnica de Ambato, Ambato, Ecuador, tdj.paredes@uta.edu.ec

2 Universidad Técnica de Ambato, Ambato, Ecuador, al.jimenez@uta.edu.ec

3 Universidad Técnica de Ambato, Ambato, Ecuador, alenakquirogal@uta.edu.ec

${ }^{4}$ Universidad Técnica de Ambato, Ambato, Ecuador, angelppoaquizap@uta.edu.ec 
of the Research Unit of the Ambato Technical University. The findings lead to new approach to deal with families communication, parental skills and community leaders involvement.

Keywords: Violence, Children, Adolescents, Parents, Community, Education.

\section{Resumen.}

Esta investigación se centra en averiguar la manera en que padres, niños y líderes comprenden y conceptualizan la violencia. El artículo explora cómo se comunican las familias, las habilidades de los padres y la influencia de los líderes comunitarios. El proyecto se realizó en la localidad de Pelileo, Ecuador, considerada área rural, con una muestra de $(n=260)$ entre los que se encontraban 59 líderes comunitarios, 110 niños y adolescentes y 101 padres. Los individuos pertenecían a las poblaciones de Pelileo, Bolívar, Huambaló y Cotaló, pequeños pueblos rurales que pertenecen al estado de Tunguahua, Ecuador. Para obtener datos se realizó una investigación cuantitativa utilizando encuestas para cada grupo. La investigación siguió las normas y principios de la Unidad de Investigación de la Universidad Técnica Ambato. Los resultados confirmaron la presencia de abuso físico, emocional y sexual contra niños en esta área rural donde los actores sociales solicitaron un componente de educación en las áreas de habilidades parentales y comprensión de la violencia. Como conclusiones de esta investigación se dedujo que era necesaria la cooperación con unidades gubernamentales, como el Departamento de Educación, la Junta Cantonal y la Universidad, para ofrecer el componente educativo solicitado por los actores sociales que formaron parte del proceso investigativo.

Palabras Claves: Violencia, niños, adolescentes, padres, comunidad, educación

\section{Introducción.}

Las variables de investigación son la violencia contra los niños y en examinar aquellas prácticas culturales que pueden reducir o prevenir esta violencia. El documento describirá a la familia en relación con la violencia, la comunicación y las habilidades de los padres, además de una descripción de la participación de los líderes comunitarios en este tema.

El problema de la violencia contra menores es global, y Ecuador no está exento de esto. La presente investigación abordará este problema a partir de la influencia de la variable de prácticas culturales de esta comunidad en los actores sociales Esta variable es muy compleja, por lo que se centrará en los siguientes aspectos: El aprendizaje de modelos con roles comportamentales, como la aceptación cultural del machismo, el uso del poder contra menores, la influencia de la iglesia en la perpetuación de las asignaciones de identificación de género, la comunicación entre padres e hijos, la transmisión de violencia simbólica entre 
generaciones y la influencia de los individuos en la comunidad que tienen roles autoritarios y que aceptan la violencia en sus contextos.

Existe una correlación entre la violencia contra las mujeres y la presente investigación debido al hecho de que los niños están casi siempre presentes en los hogares cuando estos maltratos se producen. Según el Instituto Nacional de Estadística y Censos INEC, (2012, pág. s/p), Ecuador informó el 90\% de las mujeres en el país están expuestas a la violencia y no abandonan a sus parejas. Tungurahua donde se realizó la investigación es el segundo estado del país con mayor índice de violencia contra las mujeres, por lo que se considera que esta investigación es necesaria ya que los niños presencian estos tipos de maltratos y es urgente averiguar cómo se ven estas familias y que servicios se les puede brindar.

\section{Familia.}

La conceptualización ha estado cambiando a través de los tiempos, manteniendo algunas de sus raíces de lo que se consideraría como "familia tradicional" pero también añadiendo excepciones a este concepto. Por ejemplo Morris et al. (2015, pág. 51) indica que, para hablar de familia, es más útil concentrarse en las prácticas familiares que en su terreno conceptual, ya que tales miríadas de conceptualizaciones se presentan como reflejo de contextos socioculturales, económicos, políticos, temporales y espaciales. Basandose en esta idea esta investigación se ha centrado en el estudio de las practicas familiares a través de observar cómo estas se comunican en el seno familiar y con su comunidad para poder extraer conclusiones de acuerdo a lo que significan las familias en el contexto tungurahuense. Morris define la opinión de la sociedad sobre quién es responsable de la violencia familiar, sobre el contexto moral en que los niños son exonerados y los padres culpables de los problemas expuestos por sus hijos.

\section{Familia y Violencia.}

La violencia familiar no es nueva para la humanidad: La sociedad tiende a pensar que las familias son una fuente de protección, a veces cuando y la violencia y el descuido están presentes, esta violencia es oculta o ignorada o no se reconoce como abuso y no se denuncia. Las raíces de este problema son cuestiones estructurales de la familia, tales como el tiempo que la familia pasa juntos, la relación de poder entre los padres y los hijos y la subordinación de los hijos a sus propios padres que conlleva un riesgo para la victimización.

El ideal de una familia es también parte del problema, los derechos de los padres estan sobre los derechos de sus hijos. El derecho a la intimidad familiar y la auto-autonomía, la aceptación cultural de un castigo físico, la aceptación social de la violencia familiar y su glorificación son factores a tener en cuenta. Barnet et al. (2011, págs. 1-6). 
El concepto de violencia incluye (ya sean permitidos o evitados) actos de abuso físico, sexual o emocional, los cuales impiden el desarrollo saludable de los miembros de la familia (ibid). También añade en su estudio que la violencia se define como el uso intencional de la fuerza o poder físico de un acto o amenaza contra uno mismo, grupo o comunidad (2013, pág. 21), que causa o tiene la probabilidad de causar lesiones, muerte, daños o trastornos psicológicos y problemas de desarrollo. Humphreys y Zeanah (2014, pág. 154) en su estudio longitudinal de niños menores de 12 años, fueron entrevistados posteriormente a los 29 años, en los siguientes ámbitos: psicopatología, abuso de sustancias, nivel educativo, empleo, antecedentes penales.

Reafirmó que las experiencias adversas del niño y los resultados negativos posteriores incluyendo la psicopatología, el impacto en el cerebro y el sistema biológico de un niño en desarrollo en los siguientes dominios: conducta social, trastorno de estrés postraumático, trastorno de déficit de atención e hiperactividad y problemas de conducta resultantes de estrés y trauma. Especialmente cuando hay negligencia y abuso.

Continuando con las consecuencias perversas de la violencia (ibid) ya que los primeros 3 años de vida son vitales para el desarrollo cognitivo, afectivo y social de los niños, el cuidado de los cuidadores ayuda a los bebés a regular su respuesta física y emocional a los estresores. Los cuidadores ausentes o negligentes no dan las experiencias interactivas necesarias que ayudan a las habilidades del niño desde el desarrollo del lenguaje a la competencia social. Efectos perjudiciales pueden ser los resultados neurobiológicos y conductuales específicos. La exposición a la violencia se ha asociado con la longitud más corta de los telómeros, el abuso físico vinculado a los cambios en la metilación del ADN, ya que varias veces en el gen del receptor de glucocorticoides mostró metilación aumentada en los jóvenes que han sido abusos en comparación con los niños no abusados. Los niños que experimentaron abuso físico antes de los 5 años tenían cuatro veces más probabilidades de tener problemas de conducta en el 3er y 4to grado. El comportamiento de los padres se ha relacionado con el inicio y el mantenimiento de los comportamientos agresivos y delincuentes de los niños. Hurt y Betancourt (2015, pág. 148) añade que la exposición temprana a la pobreza y a entornos tóxicos que incluyen la presencia de violencia y abuso infantil, provoca retrasos en el desarrollo del lenguaje, el desempeño cognitivo y escolar recomendando la intervención temprana en bebés y niños pequeños para prevenir retrasos en su desarrollo.

Conocer el grado de las consecuencias de la violencia familiar son los intentos de suicidio, Haglund, A et al. (2016, pág. 1), en su estudio de los intentos de suicidio concluyen que la evaluación de la violencia interpersonal puede ser de valor en la evaluación del riesgo después de un intento de suicidio. Un intento de suicidio es el mayor riesgo de suicidio. La exposición a la violencia doméstica se asocia a un intento de suicidio posterior tanto para la 
víctima como para el perpetrador; La investigación utilizó el KIVS para medir expresiones de violencia y violencia como un niño (EDAD 6-14). El estudio también mostró correlación entre los niños expuestos a la violencia doméstica y estar expuestos a la intimidación.

Las leyes continúan siendo una solución a este problema, pero no se demuestra que ayuden a su reducción. Estadísticas de New Jersey donde el autor trabajó en protección infantil: USA Supporting Strong Families en Nueva Jersey (2012, pág. s/p) informó que en 2012 9250 niños fueron víctimas de abuso y negligencia en un rango de 0.79 por cada 100.000 niños. La tasa nacional de muertes es de 2.20 por 100.000 niños. (Departamento de Servicios Humanos de los Estados Unidos, 2012).

A esta realidad podemos concluir que en países con sistemas y protocolos avanzados en protección de la infancia persisten las tasas de incidencia de abuso y aún más en países con protocolos insipientes.

Los números en Ecuador también son discursos, Desafíos (2013, pág. 15) En Ecuador los niños crecieron con factores físicos, intelectuales, sociales y económicos no favorables, en su estudio sobre abuso contra niños declaró que el 51\% de los niños denunciaron abuso dentro de sus familias, 7 de 10 reportaron abuso físico, sexual y negligencia.

En Ecuador, según la Agencia de Noticias Público de Ecuador y Sudamérica en 2014, 271 denuncias de abusos sexuales fueron reportadas en escuelas. En 2013 de las 634 denuncias de violencia sexual contra niños. Sólo se investigó el 6\% (ONU, 2014).

La defensa ecuatoriana de los derechos sexuales y la reproducción de Ecuador (2011) la tasa de suicidio entre las niñas edad tienen correlación directa con los embarazos que los resultados de la violencia sexual. En 2010, 10 denuncias de abuso sexual diarias para niñas entre 12 y 14 años de edad, habían aumentado en un $78 \%$ en los últimos diez años. Ahora una de cuatro mujeres ha sido víctima de violencia sexual, estamos hablando de que un millón de mujeres en Ecuador habían sufrido violencia sexual antes de los 18 años de edad. (INEC 2011).

El aspecto socioeconómico de este problema. (2016) Ecuador $8.56 \%$ de niños y adolescentes de 5 a 7 años trabajan. $62.8 \%$ son niños y $37.2 \%$ son niñas. El $60 \%$ trabaja para ayudar a sus familias. 8.6\% de niños y adolescentes que trabajan el $56 \%$ lo hacen en actividades consideradas peligrosas. El 12,6\% de los niños y adolescentes que se quedan en casa para hacer tareas no asisten a la escuela. 
Paredes, (2015, pág. 35) en su proyecto de investigación en una escuela en Ambato encontró que el $20 \%$ de los niños sufrieron abuso físico, el 35\% reportaron abuso emocional.

\section{Comunicación Familiar y Habilidades Parentales.}

Una buena comunicación es básica para construir buenas familias, no sólo les ayudó a solucionar sus problemas. Pero para buscar respuestas tenemos que entender que quieren esta ayuda en el camino de los planes de la familia eran ellos minded. (Morris ibid, página 58). Por lo tanto, la investigación se centra en la obtención de datos sobre qué y cómo esto podría traducirse en servicios adecuados para ellos.

Muchos factores influyen en la comunicación familiar, los padres y sus capacidades parentales, la historia de abuso, la edad, la educación, las madres solteras, los cuidadores no biológicos, el aislamiento social, la desorganización y la violencia en el mundo. Mala relación entre padres e hijos. Kliksberg (2009, pp. 1-3) expresa la influencia negativa de la comunidad, los barrios donde hay violencia, pobreza y desempleo, que alientan el constante movimiento de familias, además de la exposición y facilidad que estas personas están expuestas al uso y venta de drogas.

\section{Líderes Comunitarios Sobre la Violencia Contra los Niños.}

Un orden social da confianza, por lo tanto, es importante saber cómo piensan y actúan los líderes comunitarios sobre la violencia en sus comunidades. Intravia, J; Stewart, E; Warren P y Wolff (2016, pág. 1) informaron que una comunidad que confía en que la gente alrededor de ellos vive en un alto orden y son menos temerosos de su ambiente, de lo contrario pueden ver a la policía menos sensible socavar la confianza de las personas. Como se ha citado anteriormente, las familias responden a sus contextos socioculturales y políticos. Este artículo analiza cómo los líderes de la comunidad donde se desarrolló la investigación piensan y actuaron sobre los diferentes enfoques de la violencia contra niños y adolescentes. Es bueno indicar que dos nuevas tesis para investigar la influencia de la policía y la reducción de la violencia ha salido de esta investigación.

\section{Metodología.}

\section{Participantes.}

La muestra $(\mathrm{n}=260)$ está compuesta por 59 líderes comunitarios, 110 niños y 101 padres, todos ellos habitantes de Pelileo, Bolívar, Humbaló y Cotaló, pertenecientes a la provincia de Tungurahua (Ecuador). Los estudiantes de la carrera de Trabajo Social cooperaron tomaron la encuesta, la misma que se capacitó en el proceso de la investigación, para justificar la buena aplicación de la encuesta, que tomó unos diez minutos para cada participante. Los datos fueron analizados con SPSS 23 (versión ventanas 32 bits). Los participantes fueron informados y su participación fue voluntaria. 


\section{Instrumentos.}

Se utilizó una encuesta para cada grupo, las encuestas fueron utilizadas por el proyecto de construcción y fortalecimiento de la comunidad basada en ANPPCAN (2013) encuesta infantil, se utilizó un modificado ISPCAN Child Abuse Screening Tool (ICAST). Estas encuestas consistían en preguntas abiertas y cerradas dependiendo del tipo de información que se pretendía extraer.

\section{Procedimiento.}

Una vez que se determinó el tamaño de la muestra y la cuota para cada estudio, se pidió permiso a las personas e instituciones correspondientes. Para la realización del trabajo de campo se consideró la colaboración de estudiantes pertenecientes a un curso de la carrera de Trabajo Social, los cuales fueron entrenados para asegurar la correcta administración de los cuestionarios, con una duración media de la encuesta de 10 minutos para cada grupo. En todos los casos, los sujetos que participaron estuvieron adecuadamente informados del carácter voluntario y anónimo de su participación en el estudio y de los objetivos del estudio, aceptando voluntariamente participar sin recibir ninguna compensación por ello.

La investigación cumplió con las normas establecidas por el Comité de Ética en Investigación y Enseñanza de la Universidad Técnica de Ambato.

Los datos recogidos fueron analizados por el programa estadístico SPSS (versión 23 para Windows 32 bits).

\section{Resultados.}

\section{Líderes Comunitarios.}

La mayoría afirmó realizar actividades educativas para prevenir o reducir los abusos contra niños $(85 \%)$ y ofrecer la ayuda que pudieran en estos casos, ya sea a veces (33\%) o siempre (67\%), siendo el asesoramiento el método más utilizado. Una gran parte coincide en que la familia debe ser la principal responsable de resolver el problema del maltrato infantil, también comentó que sólo el 30\% no aceptó intervenciones familiares, que consistían en el uso de charlas, talleres y visitas a psicólogos. $75 \%$ negaron que las leyes contra el abuso infantil fueran la única solución al problema, argumentando que el tema debía tratarse desde una perspectiva más holística, aumentando la conciencia de la sociedad, trabajando especialmente con las familias y la necesaria participación de la comunidad y la comunidad. La policía para reducir la violencia contra menores.

También el 45\% dijo que había conocido casos de niños que habían sufrido abuso sexual, de los cuales el $75 \%$ decidió reportarlo a instituciones como el consejo cantonal, la policía 
u otros líderes comunitarios, y fue efectivo en la mayoría de las ocasiones. A su vez, el 35\% estuvo de acuerdo en la existencia de tradiciones culturales que podrían ser consideradas como abusos, como adultos casados con menores o castigo. En cuanto a la última, propusieron medidas disciplinarias alternativas como discutir o explicar a sus hijos lo que han hecho mal y retirar los privilegios como un método de castigo antes de recurrir a la violencia.

Las correlaciones de Spearman más significativas estadísticamente ( $p$ <.01) mostraron que entre los mayores de estos líderes, mayor era la aceptación de las intervenciones a las familias $(\mathrm{r}=.663)$. También se encontró que aquellos que más valoraban la importancia de la comunidad eran los que tenían más casos conocidos de niños que habían sufrido abuso sexual $(r=.597)$.

\section{Niños.}

La edad promedio fue de 14,56 años, con un grado académico de 8,3 como calificación promedio. Más del $60 \%$ reportó que a veces eran castigados físicamente por malas calificaciones.

El $80 \%$ vivía con padre y madre, mientras que el $18 \%$ vivía solo con su madre. En cuanto al grado académico de estos, el 50\% de los padres tenía la primaria, seguida por el $30 \%$ con el título de secundaria; mientras que las primarias correspondieron a ellas en el $60 \%$ de los casos y la realización de la secundaria en el 22\%, mostrando así diferencias académicas según género.

El 84\% afirmó que asistía a la escuela mucho o siempre, mientras que el 15\% hacía poco o nada, aludiendo a razones tales como insatisfacción y especialmente tener que trabajar para conseguir dinero.

La mitad de ellos admitió que a veces habían recibido castigo corporal cuando se portaron mal, causándoles tristeza, llanto y dolor. Al 50\%, sus cuidadores habían sido llamados vagos o tonto en algunas ocasiones, mientras que el $30 \%$ había sido a veces degradado, aterrorizado o ignorado, admitiendo que la agresión física era lo que menos les gustaba.

Por otra parte, el 99\% consideró que el alimento, el refugio, la salud y la educación proporcionados por sus cuidadores eran suficientes o buenos, argumentando que generalmente tenían todo lo que necesitaban.

El 84\% tenía diálogo y explicaciones como un método disciplinario más justo que debería ser usado por sus padres en caso de regañar, mientras que el 14\% optaría por la retirada de privilegios. Sin embargo, cuando se les preguntó qué usaban sus familiares, el 10\% dijo que la violencia era el método más común. 
Como resultado, casi el 30\% informó que a veces fueron testigos de violencia doméstica en sus hogares, sintiéndose mal de una situación que les causó miedo y tristeza, y considerando que podría conducir a consecuencias como baja autoestima, malas calificaciones, ira, depresión, mal comportamiento y otras implicaciones para el abuso físico y emocional.

A diferencia de los líderes comunitarios, alrededor del $70 \%$ no conocía a otros niños que habían sufrido abuso físico, bajando esta cifra al $60 \%$ en el caso de abuso emocional y aumentando al $94 \%$ cuando no conocían a niños que habían sufrido algún tipo de abuso sexual.

En este último aspecto, el 30\% de ellos afirmó que rara vez podían distinguir entre un tacto apropiado y otro que no lo era. Además, el $10 \%$ informó de que a veces se intentó tocar indebidamente, reaccionando de diversas maneras, como informar, detener o decirlo. Como una figura para recurrir al abuso, el 50\% prefiere la madre, mientras que el padre el 10\%, casi lo mismo que los amigos.

Esta diferencia de confianza entre los padres también se refleja en el nivel de comunicación con ellos. Mientras que el $50 \%$ dice que es bueno para su padre y amigos, el $75 \%$ siente lo mismo de su madre, esto muestra una preferencia materna.

Por último, el $40 \%$ se molestó por el hecho de que sus padres estaban preguntando dónde estaban cada vez que salieron privándolos de su libertad como un acto de falta de respeto.

En cuanto a las correlaciones significativas ( $\mathrm{p}<0,05)$, se informó que las niñas habían sido llamadas vagas o tonto en menor medida que los niños $(r=-234)$. Al mismo tiempo, el género también tuvo un impacto en el posible abuso de cualquier tipo, donde optaron por igual para ambos padres; Mientras que se recaería sobre todo en la madre (65\%) y menos en el padre $(7 \%)$, como persona confidente.

Se encontró que a una edad más avanzada se les aplicaba menos castigo corporal $(\mathrm{r}=-235)$, pero más habían intentado tocarlos inapropiadamente $(\mathrm{r}=0,237)$.

Se observó a través del número de hermanos, ya que las madres que tenían más hijos también eran aquellas que tenían un grado académico más bajo y viceversa $(r=-278)$. El nivel de educación y cualificación de los encuestados se correlacionó positivamente con el de ambos padres y asistiendo a la escuela. Sin embargo, lo hizo de manera negativa con el hecho de que sus padres los observaban cuando se iban, haciéndolo en menor medida a aquellos con educación académica superior. 
Cuanto más nivel de educación tenía el padre, mayor era la madre $(r=.697)$ y menos violencia doméstica en el hogar $(r=-.219)$. Al mismo tiempo, prestar más atención en la escuela estaba relacionado con una buena comunicación con los hermanos $(\mathrm{r}=.318)$ y molestarlo menos que mirarlo al salir de casa $(\mathrm{r}=-251)$.

Se observó que aquellos que habían sido sometidos a castigos corporales cuando estaban enfermos, también consideraron que los recursos dados por sus padres eran escasos o insuficientes $(r=-294)$. Además, el hecho de que se les llamó tonto o vago correlacionado con el castigo físico por traer malas marcas $(r=.274)$. En estos casos de violencia psicológica verbal hubo peor comunicación paterna $(r=-.370)$, maternal $\mathrm{R}=-.275)$ y fraternal $(\mathrm{r}=-.309)$.

\section{Padres.}

La edad media era de 35 años, que tenía dos o más hijos. El ingreso mensual promedio fue de $\$ 434$ mensuales. $50 \%$ trabajaban fuera de casa y casi $20 \%$ vivían con familias extensas. Similar a los niños, el 30\% reportó casos de abuso físico, 50\% emocional y $15 \%$ sexual, con las mismas implicaciones emocionales que se mencionaron anteriormente. De los que alguna vez conocieron algún tipo de abuso contra menores, reportaron casi el $70 \%$, especialmente a los familiares de la víctima, a la junta cantonal de la policía.

Teniendo en cuenta que algunos de los padres encuestados también eran líderes comunitarios, se encontró una opinión similar con respecto al $20 \%$ de las tradiciones culturales consideradas abusivas, especialmente aquellas relacionadas con matrimonios entre adultos con menores de edad.

El 30\% consideró que las mujeres ocasionalmente provocan episodios de violencia contra sí mismos por razones tales como celos, provocando al marido, no haciendo su trabajo o estar lejos de casa más de lo que deberían. Esto explicaría en parte el 35\% que afirmó haber sufrido episodios de violencia doméstica en el hogar, en su mayoría psicológicos. Sin embargo, la proporción de violencia doméstica presenciada por los niños disminuye al $25 \%$, lo que demuestra que los niños no siempre están involucrados en estos casos.

De los padres encuestados, el $20 \%$ pasaba de cero a una hora diaria a sus hijos para jugar, leer, etc., 30\% de una a dos horas y 50\% más de dos horas. Más del $90 \%$ afirmó preguntarle a sus hijos cómo iban las clases y otras actividades, el 90\% admitió que explicaba los errores cometidos cuando sus hijos eran malos en la mayoría de las ocasiones, el 70\% les hablaba de asuntos sexuales más de una vez al mes cuyos hijos eran viejos Suficiente para ello, y sólo una persona dijo que nunca podrían satisfacer económicamente las necesidades de sus hijos. 
El 98\% utilizó medios disciplinarios alternativos al castigo corporal, tales como retirar los privilegios (60\%) o explicar lo que habían hecho mal (39\%).\}

El $40 \%$ afirmó que no debían ser castigados físicamente, mientras que el resto lo había hecho en varias ocasiones, justificándose a sí mismos con la reprimenda de la rebelión de los niños al final y que solo ellos pueden castigarlos de esa manera, ya sea el padre o la madre Proporción igual dada el caso).

El 58\% nunca había conocido a niños víctimas de abuso emocional, y sólo tres personas reconocieron no saber con quién estaban sus hijos cuando salieron de casa, o dejándolos solos por la noche, ya que el resto consideró peligroso hacerlo.

El 90\% consideró que afectó a sus hijos a presenciar episodios de violencia doméstica, especialmente en su estado psicológico. Al mismo tiempo, el $45 \%$ consideró que la reacción de la policía a estos episodios era inadecuada o no era útil.

Por último, el 99\% quisiera que las escuelas y los GAD ofrecieran talleres para padres, especialmente sobre cómo educar a los niños.

En relación con las correlaciones más significativas ( $\mathrm{p}<.05)$, las mujeres trabajaron menos fuera del hogar $(r=-290)$, recibieron un pago menor $(r=-261)$, lo que fue peor para las necesidades económicas de sus hijos. 216) y hablaban menos de sexualidad con ellos ( $\mathrm{r}=-$ 278).

A una edad avanzada se encontró que se pasaba menos tiempo con los niños a leer o jugar con ellos $(\mathrm{r}=-308)$, pero se les preguntó más acerca de cómo se fue la escuela u otras actividades $(\mathrm{r}=.219)$, así como hablar con ellos Sobre temas sexuales $(\mathrm{r}=.326)$.

Un alto nivel educativo se correlacionó con mayores ingresos mensuales $(r=.303)$, conociendo a los niños sexualmente abusados $(\mathrm{r}=.312)$, sabiendo con quién estaban sus hijos cuando salieron de casa $(\mathrm{r}=.236)$ ya un mayor grado de $(\mathrm{R}=.286)$, pero negativamente con sus hijos que tuvieron menos episodios de violencia doméstica $(\mathrm{r}=$ 240), preguntándoles cómo estaban en la escuela u otras actividades $(\mathrm{r}=-279)$ y un número menor de niños $(r=-355)$.

Finalmente, el hecho de experimentar episodios de violencia doméstica estuvo relacionado con explicar menos los errores cometidos a los niños $(r=-226)$, empeorarlos por no comprometerse con sus necesidades económicas $(r=-274)$ y menos colaboración $(\mathrm{R}=-$ 667). 


\section{Conclusiones.}

- Al mismo tiempo, el grado de culpabilidad encontrado en las mujeres es a menudo llamado la causa de episodios de violencia por razones relacionadas con el incumplimiento de su supuesto papel de mujer ama de casa. También sería de destacar el desacuerdo de algunas tradiciones como el matrimonio de adultos con menores, así como la violencia física como castigo disciplinario a menores, algunos de los cuales mostraron una insatisfacción por parte de la escuela mientras estaban ausentes por razones laborales o económicas. La necesidad de que los actores sociales entiendan que el alto porcentaje de niños que reciben abuso físico y sexual infantil debe ser abordado y reducido, abuso que fue admitido por sus padres.

- De los resultados podemos concluir la importancia de la comunidad en casos de abuso de menores, así como una buena conciliación familiar en la educación tanto académica como de valores. Además, existe una preocupación por las leyes y la intervención policial en los temas de abuso infantil, al considerarlos insuficientes en muchas ocasiones. Este resultado condujo a un servicio de talleres para las familias sobre estos temas. Por último, las familias con mayor nivel académico y mayor ingreso mensual fueron las que tuvieron la mejor relación con sus hijos, así como aquellas con los menores episodios de violencia doméstica.

- Wessels $(2015$, p.8) cree que para desarrollar y mejorar las metodologías para prevenir o reducir el abuso infantil, los actores sociales involucrados deben estar directamente involucrados, y se necesita una investigación profunda de las raíces de una comunidad y determinar si en una cultura Con sus indicadores en el aprendizaje, la comunicación y la influencia de personas importantes en la comunidad crea este problema.

- La investigación también impulsó la creación de una Guía trabajada con un enfoque interdisciplinario. Los objetivos son: (2016)

Indicar a los profesionales que trabajan en las instituciones encargadas de la protección de la infancia.

Procedimientos de servicios para aquellos profesionales e instituciones.

Procedimientos metodológicos para el trabajo con profesionales fuera de las instituciones públicas.

Educación de las comunidades para prevenir la violencia doméstica.

Un objetivo es prevenir el abuso sexual contra los niños mediante talleres impartidos por psicólogos y trabajadores sociales. Que les enseñan cuatro habilidades básicas:

- Enséñeles a decir "no", necesitan reconocer que pueden disputar algo y decir no en ocasiones especiales.

- Enséñeles a identificar el abuso: Deben saber que dentro de la familia y otras relaciones existen buenos y malos comportamientos. Esto debe ser claro para no animar a tener miedo de un buen contacto.

- Enséñeles a confrontar situaciones: cuando es posible deben detener al agresor, en lugares y momentos que no arriesguen sus vidas.

- Rompa el ciclo del silencio: Enséñeles a no guardar el secreto, en caso de abuso. (Save the Children, 1998) 
- El componente educativo que inició el proyecto de investigación ha sido bien recibido por los actores sociales, la comunidad ha respondido favorablemente a los discursos de radioeducación impartidos por los profesionales y los talleres educativos en las escuelas para niños menores donde el abuso tenía tasas más altas.

- Los resultados de esta investigación también conducirán a la redacción de un protocolo institucional para tratar casos de abuso infantil que tenga en cuenta las familias, sus necesidades y fortalezas.

\section{Referencias bibliográficas.}

Annie Casey Foundation. (2013). Mapa Mundial de la Familia. Trends Child, 72.

Barnett, O., Miller-Perrin, C., \& Perrin, R. (2011). Family Violence (3ra ed.). (T. Paredes, Trad.) Thousand Oaks, California, USA:

Sage.Heinz, A., Beck, A., Meyer-Lindenberg, A., Sterzer, P. and Heinz, A. (2011). Cognitive and neurobiological mechanisms of alcohol-related aggression. Nature Reviews Neuroscience, 12(7), pp.400-413.

Group of Defense Of Sexual Rights and Reproduction. (2011)

Haglund, A., Asa, L., Henrik, L., Salander, Salander Renberg, E., Jussi, J., y otros. (14 de November de 2016). Interpersonal violence and the prediction of short-term risk of repeat suicide attempt. Scientific Report, 6(36892), 1-8.

Humphreys, K., \& Zeanah, C. (6 de Agosto de 2014). Deviations from the expectable enviroment in early childhood and emerging Psychopathology. (A. C. Neuropsychopharmacology, Ed.) Neuropsychopharmacology Reviews, 40, 154170.

Hurt, H., \& Betancourt, L. (16 de Diciembre de 2015). Effects of socioeconomic status disparity on child language and neural outcome: how early is early. Pediatric Research, 148-158.

Revistavirtual. (2016). Recuperado el 25 de April de 2017, de http://revistavirtual.ucn.edu.co/index.php/RevistaUCN/article/view/270/510R

INEC. (2012). Quito.

Intravia, J., Stewart, E., Warren, P., Warren, P., \& Wolff, K. (2016). Neighborhood disorder and Generalized trust: A multilevel mediation of social mechanisms. Journal of Criminal Justice, 46, 148-158.

Jagannathan, M. (2013). Protecting Children in the age of outrage. A new perspective on Child Protection Services. Child Protection Services.

Kliksberg, B. (2009). La Familia en América Latina. XIX Congreso Panamericano del niño, niña y adolescentes con la Familia., 30.

Ministerio de Inclusión Económico y Social. (2016). inclusion.gov. Recuperado el 25 de Abril de 2017, de http://www.inclusion.gob.ec/se-presentaron-los-resultados-de-laprimera-encuesta-de-trabajo-infantil-en-ecuador/ 
Morris, K., White, S., Doherty, P., \& Warkis Lisa. (August de 2015). Out of time: theorizing family in social work practice. Child and Family Social Work, 51-60.

ONU. (2014). Agencia de Noticias de Suramérica ANDES.

Paredes, T. (2015). El legado alcohólico de los padres y los problemas educativos de sus hijos adolescentes estudiantes del Colegio Tirso de Molina de Ambato. Ambato: Universidad Técnica de Ambato.

UNICEF. (8 de Julio de 2013). Maltrato infantil, una dolorosa realidad. Desafios, 20.

US Department of Human Services. (2012).

Wessells, M. (9 de Abril de 2015). Buttom-up approaches to strengthing child protection systems: Placing families and communities at the center. Child Abuse, 25.

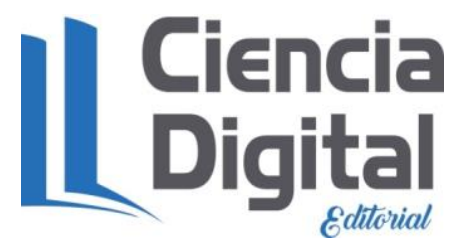


Para citar el artículo indexado.

Paredes T, Jiménez A., Quiroga M. \& Poaquiza P. (2018). La violencia contra niños, niñas y adolescentes, la línea entre el abuso y la disciplina. Revista electrónica Ciencia Digital 2(2), 155-169. Recuperado desde: http://cienciadigital.org/revistacienciadigital2/index.php/CienciaDigital/article/view/79/74

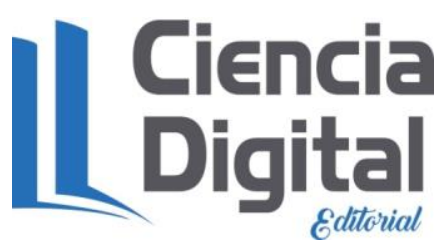

El artículo que se publica es de exclusiva responsabilidad de los autores y no necesariamente reflejan el pensamiento de la Revista Ciencia Digital.

El articulo queda en propiedad de la revista y, por tanto, su publicación parcial y/o total en otro medio tiene que ser autorizado por el director de la Revista Ciencia Digital.
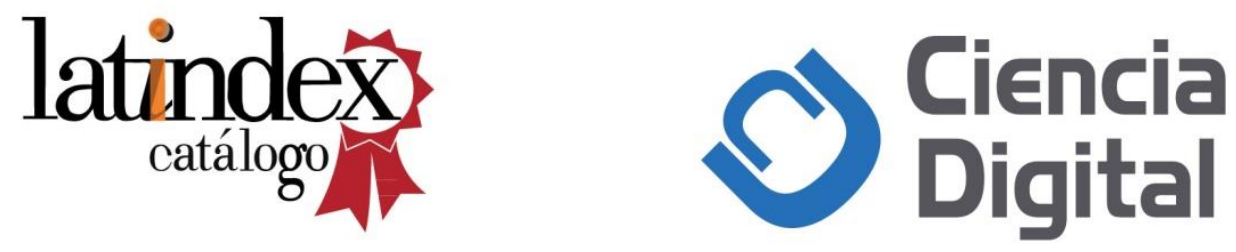